\title{
Genus sets and SNT sets of certain connective covering spaces
}

\author{
by \\ Huale Huang (Morristown, NJ, and Warren, NJ) and \\ Joseph Roitberg (New York, NY)
}

\begin{abstract}
We study the genus and SNT sets of connective covering spaces of familiar finite $\mathrm{CW}$-complexes, both of rationally elliptic type (e.g. quaternionic projective spaces) and of rationally hyperbolic type (e.g. one-point union of a pair of spheres). In connection with the latter situation, we are led to an independently interesting question in group theory: if $f$ is a homomorphism from $\operatorname{Gl}(\nu, A)$ to $\operatorname{Gl}(n, A), \nu<n, A=\mathbb{Z}$, resp. $\mathbb{Z}_{p}$, does the image of $f$ have infinite, resp. uncountably infinite, index in $\operatorname{Gl}(n, A)$ ?
\end{abstract}

1. Introduction and statement of results. In this paper, we study the genus sets and SNT sets of certain $m$-connective covering spaces $X\langle m\rangle$, following the work initiated by McGibbon and Møller ([16]), and continued by McGibbon and Roitberg ([17]). Before stating our main results, we recall the basic notions; in the following definitions, $X$ and $Y$ are assumed to be spaces of the homotopy type of nilpotent, finite type CW-complexes.

DEFINITION 1.

(i) $\widehat{\mathcal{G}}(X)$ is the set of homotopy types of spaces $Y$ such that the profinite completion $\widehat{Y}$ of $Y$ is homotopy equivalent to the profinite completion $\widehat{X}$ of $X$. (Note that $\widehat{X}$ is canonically homotopy equivalent to the product $\prod X_{p}$, where $X_{p}$ is the $p$-completion of $X$.)

(ii) $\widehat{\mathcal{G}}_{0}(X)$ is the subset of $\widehat{\mathcal{G}}(X)$ for which the rationalizations $X_{(0)}, Y_{(0)}$ of $X, Y$ are homotopy equivalent.

(iii) $\mathcal{G}(X)$ is the subset of $\widehat{\mathcal{G}}_{0}(X)$ for which the $p$-localizations $X_{(p)}, Y_{(p)}$ of $X, Y$ are homotopy equivalent for all primes $p$.

Thus we have the set-theoretic inclusions

$$
\mathcal{G}(X) \subset \widehat{\mathcal{G}}(X) \subset \widehat{\mathcal{G}}(X) .
$$

2000 Mathematics Subject Classification: 55P60, 55P62, 55P15, 20 G99.

Key words and phrases: $m$-connective covering, completion genus, Mislin genus, automorphism group of a space, Neisendorfer's localization theorem, basic Whitehead product, general linear group over the integers or the $p$-adic integers. 
$\widehat{\mathcal{G}}(X)$ is called the completion genus of $X$ and $\mathcal{G}(X)$ is called the localization genus or Mislin genus of $X$.

Definition 2. $\operatorname{SNT}(X)$ is the set of homotopy types of spaces $Y$ such that the $m$ th Postnikov approximations $P_{m}(X), P_{m}(Y)$ are homotopy equivalent for all positive integers $m$.

In [30], Wilkerson proves that the completion genus set of a 1-connected, finite CW-complex, or finite Postnikov space, is finite. (Hence the same is true of the other two genus sets.) Wilkerson's result is certainly not valid for general 1-connected, finite type $\mathrm{CW}$-complexes. As a simple example, let $C_{\alpha}=S^{r} \cup_{\alpha} e^{n}, r>1$, be the mapping cone of a homotopy element $\alpha$ in the stable range and of order a prime $p>3$, and let $X$ be the one-point union $\bigvee_{i=0}^{\infty} \Sigma^{n_{i}} C_{\alpha}$, where $n_{0}=0$ and $n_{i}$ is chosen so that $n_{i}+r>n_{i-1}+n$. As $\mathcal{G}\left(\sum^{n_{i}} C_{\alpha}\right)$ has cardinality $\frac{1}{2}(p-1)>1$ ([10; III, Example 1.3]), it is not difficult to verify that $\mathcal{G}(X)$ is uncountably infinite.

An interesting example of an uncountably infinite Mislin genus set, due to Rector ([23]) for the case $G=S^{3}$ and to Møller ([20]) for all nontrivial, 1-connected, compact Lie groups $G$, is $\mathcal{G}(B G)$, where $B G$ is the classifying space of $G$. Another striking, and somewhat surprising, example of an uncountably infinite Mislin genus set is given in the paper [16] by McGibbon and Møller. They prove that the Mislin genus set of $S^{2 n}\langle 2 n\rangle$, the $2 n$-connective covering space of the $2 n$-dimensional sphere $S^{2 n}, n>1$, is uncountably infinite, relying on the following corollary to a remarkable theorem of Neisendorfer ([22]): If $X$ and $Y$ are finite CW-complexes which are $1 \frac{1}{2}$-connected (i.e. $\pi_{1}=0$ and $\pi_{2}$ is finite), then the induced map $\left[X_{p}, Y_{p}\right] \rightarrow\left[X\langle m\rangle_{p}, Y\langle m\rangle_{p}\right]$ on homotopy sets is a bijection for all primes $p$ and all natural numbers $m$. Moreover, $\alpha \in\left[X_{p}, Y_{p}\right]$ is the homotopy class of a homotopy equivalence if and only if the same is true of $\alpha\langle m\rangle$.

We seek to extend the McGibbon-Møller result to a class of spaces containing $S^{2 n}$. First observe that $S^{2 n}$ is a simple example of what rational homotopy theorists term a rationally elliptic space ([6]), i.e., a 1connected, finite $\mathrm{CW}$-complex with only finitely many non-zero rational homotopy groups. Indeed, $S^{2 n}$ is a "2-stage" rationally elliptic space, with $\pi_{2 n} \otimes \mathbb{Q}=\mathbb{Q}=\pi_{4 n-1} \otimes \mathbb{Q}$ the only non-zero rational homotopy groups. The cohomology ring has the form $H^{*}\left(S^{2 n} ; \mathbb{Q}\right)=\mathbb{Q}[a] /\left\langle a^{2}\right\rangle$, the truncated polynomial ring with $\operatorname{deg}(a)=2 n$, and the Sullivan minimal model of $S_{(0)}^{2 n}$ is $\left(v, w: d w=v^{2}\right)$ with $\operatorname{deg}(v)=2 n, \operatorname{deg}(w)=4 n-1$. We will consider more general 2-stage rationally elliptic spaces, namely spaces $T$ for which $H^{*}(T ; \mathbb{Q})=\mathbb{Q}[a] /\left\langle a^{k}\right\rangle$ with $\operatorname{deg}(a)=2 n, k>1$. This rational cohomology condition implies that $T_{(0)}$ has a Sullivan minimal model of the form $\left(v, w: d w=v^{k}\right)$ with $\operatorname{deg}(v)=2 n, \operatorname{deg}(w)=2 k n-1$, hence that the two non-zero rational homotopy groups of $T$ are $\pi_{2 n} \otimes \mathbb{Q}=\mathbb{Q}=\pi_{2 k n-1} \otimes \mathbb{Q}$. 
Examples of such $T$ are:

(i) $S^{2 n}$;

(ii) $J_{k-1}\left(S^{2 n+1}\right)$, the $(k-1)$-st stage of the James reduced product construction on $S^{2 n+1}, k<\infty$;

(iii) $\mathbb{C} P^{k-1}$, the complex projective space;

(iv) $\mathbb{H} P^{h-1}$, the quaternionic projective space;

(v) $\mathbb{O} P^{2}$, the Cayley (or octonionic) projective plane;

(vi) any finite stage of a homology decomposition of $K(\mathbb{Z}, 2 n)$.

(Note that (i) is a special case of both (ii) and (vi).)

Our first main result on genus may be stated as follows.

Theorem A. Let $T$ be a $1 \frac{1}{2}$-connected space as above. Then $\widehat{\mathcal{G}}_{0}(T\langle m\rangle)$ is uncountably infinite if $2 n \leq m \leq 2 n k-2$, and is trivial (the singleton set) if $m \geq 2 n k-1$.

The hypothesis that $T$ is $1 \frac{1}{2}$-connected implies that $n>1$. To see that this hypothesis is essential, note that Theorem A does not apply to the complex projective space $\mathbb{C} P^{k-1}$ since $\pi_{2}\left(\mathbb{C} P^{k-1}\right)$ is not finite; in fact, $\mathbb{C} P^{k-1}\langle m\rangle$ is homotopy equivalent to $S^{2 k-1}\langle m\rangle$ for $m \geq 2$, and the Mislin genus of the latter space is easily seen to be finite.

As mentioned earlier, the conclusion of [16] is that $\mathcal{G}\left(S^{2 n}\langle 2 n\rangle\right)$ is uncountably infinite, $n>1$. In fact, it is stated in [16; footnote], with scant indication of proof, that $\mathcal{G}\left(S^{2 n}\langle 2 n\rangle\right)=\widehat{\mathcal{G}}_{0}\left(S^{2 n}\langle 2 n\rangle\right)$. It is natural to wonder whether this equality remains true for the more general situation in Theorem A. We only offer the following partial result.

Addendum to Theorem A. For $T$ of the form (i), (ii), (iv) or (v), $\mathcal{G}(T\langle m\rangle)=\widehat{\mathcal{G}_{0}}(T\langle m\rangle)$.

The proof of the Addendum is heavily dependent on Theorem $\mathrm{C}$ below.

In the so-called rational dichotomy, 1-connected, finite CW-complexes which are not rationally elliptic are termed rationally hyperbolic; such spaces have infinitely many non-zero rational homotopy groups and their rational homotopy groups "grow exponentially" ([6]). A simple example of such a space is the one-point union $B=S^{k} \vee S^{l}, 2 \leq k \leq l$. Our second main result on genus is centered on this example.

Theorem B. Let $B$ be as above, $k>2$. Then there exists an integer $N_{0}$ such that for all $N \geq N_{0}, \widehat{\mathcal{G}}_{0}(B\langle N\rangle)$ is uncountably infinite.

Remark. For our choice of $N_{0}$, the condition $N \geq N_{0}$ is sufficient, but not necessary, for the conclusion of Theorem B to hold. Suitable examples will be given at the end of $\S 3$. 
Note that the uncountability result in Theorem $\mathrm{B}$ is asserted for $\widehat{\mathcal{G}}_{0}$, not for $\mathcal{G}$. We do not settle the question of whether the Mislin genus of $B\langle N\rangle$ is uncountable for $N$ as in Theorem B. Interestingly, it turns out that there are examples of 1 -connected, finite type $\mathrm{CW}$-complexes $X$ such that $\mathcal{G}(X)$ is at most countably infinite (exactly countably infinite in many cases, possibly even finite in some cases) and $\widehat{\mathcal{G}}_{0}(X)$ is uncountably infinite. Basing ourselves on some computations of Møller ([20]) and McGibbon and Møller ([14]), we will present such examples in Appendix 1.

We next turn to SNT computations. With the help of the techniques in [14] and [15], we obtain the following results.

Theorem C. If $T$ is as in the Addendum, then $\operatorname{SNT}(T\langle m\rangle)$ and $\operatorname{SNT}\left(T\langle m\rangle_{p}\right)$ are trivial for all $m$ and all primes $p$.

TheOREM D. In the notation of Theorem $B, \operatorname{SNT}(B\langle N\rangle)$ is uncountably infinite for all $N \geq N_{0}$.

In contrast to the situation for genus sets, the uncountability of $\operatorname{SNT}(Z)$ is actually equivalent to the non-triviality of $\operatorname{SNT}(Z)$, provided $Z$ is of the homotopy type of a nilpotent, finite type $\mathrm{CW}$-complex, or the $P$-localization of such a space, where $P$ is a collection of primes; see [14; Corollary 2.1].

The proofs of Theorems A, B, C and D will be carried out in the next four sections. In the situation of Theorems $\mathrm{B}$ and $\mathrm{D}$ where $k=l$, our method of proof suggests a group-theoretic question which seems to have independent interest:

Question. Let $f: \operatorname{Gl}(\nu, A) \rightarrow \operatorname{Gl}(n, A)$ be a homomorphism, $\nu<n$. Is the coset space $\mathrm{Gl}(n, A) / f(\mathrm{Gl}(\mu, A))$ infinite, resp. uncountably infinite, when $A=\mathbb{Z}$, resp. $A=\mathbb{Z}_{p}$ ?

The case $\nu \leq 2$ is pertinent to Theorems $\mathrm{B}$ and $\mathrm{D}$ and differs from the case $\nu>2$. We discuss this question in Appendix 2 .

Theorems A, B, C and D constitute an amended and expanded version of a portion of the first-named author's Ph.D. dissertation [11]. Some of the results contained in these theorems were also announced in [17] and [24].

We thank Jesper Møller for some helpful correspondence, and a number of colleagues-Pierre de la Harpe, Michel Kervaire, Marston Conder, Fred Cohen, Hans-Werner Henn, Martin Moskowitz and Raymond Hoobler-for their comments on various aspects of the material in Appendix 2.

2. Proof of Theorem A and its Addendum. The proof of Theorem A (and also of Theorem B in the next section) rests on a generalization of the technique used in establishing [16; Example 4.2]. (A very brief description of this generalization was given in [17].) The starting point is the 
double coset formula of Wilkerson ([30; Theorem 3.8]), namely

$$
\widehat{\mathcal{G}}_{0}(W)=(f . c)_{*} \operatorname{Aut}\left(W_{(0)}\right) \backslash \operatorname{CAut}\left(\left(W_{(0)}\right)^{-}\right) / r_{*} \operatorname{Aut}(\widehat{W}) .
$$

In (2.1), $W$ is a space of the homotopy type of a 1-connected, finite type CW-complex, and $\left(W_{(0)}\right)^{-}$is Sullivan's formal completion of $W_{(0)}$, which is homotopy equivalent to $(\widehat{W})_{(0)}$. Furthermore $\operatorname{CAut}\left(\left(W_{(0)}\right)^{-}\right)$is the subgroup of the full automorphism group $\operatorname{Aut}\left(\left(W_{0}\right)^{-}\right)$(also known as the group of homotopy classes of self-homotopy equivalences of $\left.\left(W_{(0)}\right)^{-}\right)$consisting of those automorphisms that induce $\widehat{\mathbb{Q}}$-module automorphisms of the homotopy groups of $\left(W_{(0)}\right)^{-}$, where $\widehat{\mathbb{Q}}=\mathbb{Q} \otimes \widehat{\mathbb{Z}}$, with $\widehat{\mathbb{Z}}$ the profinite completion of $\mathbb{Z}$. Similarly we may define $\operatorname{CAut}\left(\left(W_{p}\right)_{(0)}\right), \operatorname{CAut}\left(W_{p}\right)$ and $\operatorname{CAut}(\widehat{W})$, but $\operatorname{CAut}\left(W_{p}\right)=\operatorname{Aut}\left(W_{p}\right)$ and $\operatorname{CAut}(\widehat{W})=\operatorname{Aut}(\widehat{W})$ by standard properties of $p$-completion and profinite completion for nilpotent, finite type CW-complexes. (All occurrences of $\operatorname{Aut}\left(\left(W_{p}\right)_{(0)}\right)$ in [16] and [17] should be replaced by $\operatorname{CAut}\left(\left(W_{p}\right)_{(0)}\right.$.) The homomorphisms $(f . c)_{*}$ and $r_{*}$ are induced by formal completion $f . c$ and rationalization $r$, respectively. Analysis of $r_{*} \operatorname{Aut}(\widehat{W})$ is aided by noting the existence of a commutative diagram

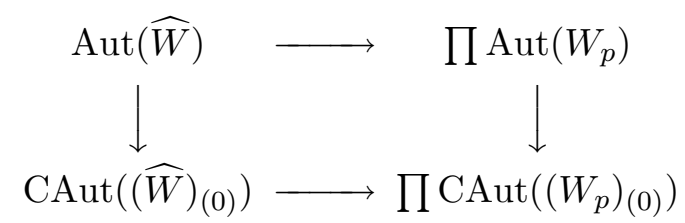

where the vertical arrows are induced by rationalization and the horizontal arrows arise from the canonical homotopy equivalence $\widehat{W} \rightarrow \prod W_{p}$ mentioned in $\S 1$; moreover, the top horizontal arrow is an isomorphism.

We apply the foregoing to $W=T\langle m\rangle$, beginning with the case $m \geq$ $2 n k-1$. In this case, all the homotopy groups of $W$ are finite. Thus $W_{(0)}$, and also $\left(W_{(0)}\right)^{-}$, is trivial, and it follows immediately from $(2.1)$ that $\widehat{\mathcal{G}}_{0}(W)$ is trivial. In the case $2 n \leq m \leq 2 n k-2$,

$$
\begin{aligned}
& W_{(0)}=K(\mathbb{Q}, 2 n k-1), \quad \text { so that } \operatorname{Aut}\left(W_{(0)}\right)=\mathbb{Q}^{*} ; \\
& \left(W_{(0)}\right)^{-}=K(\widehat{\mathbb{Q}}, 2 n k-1), \quad \text { so that } \operatorname{CAut}\left(\left(W_{(0)}\right)^{-}\right)=(\widehat{\mathbb{Q}})^{*} ; \\
& \left(W_{p}\right)_{(0)}=K\left(\mathbb{Q}_{p}, 2 n k-1\right), \quad \text { so that } \operatorname{CAut}\left(\left(W_{p}\right)_{(0)}\right)=\mathbb{Q}_{p}^{*} .
\end{aligned}
$$

In (2.3), $R^{*}$ denotes the multiplicative group of units of the ring $R$, and $\mathbb{Q}_{p}=\mathbb{Q} \otimes \mathbb{Z}_{p}$ is the field of $p$-adic numbers. Thus (2.1) reduces in this case to

$$
\mathbb{Q}^{*} \backslash(\widehat{\mathbb{Q}})^{*} / r_{*} \operatorname{Aut}(\widehat{W}),
$$

with $\mathbb{Q}^{*}$ canonically embedded in $(\widehat{\mathbb{Q}})^{*}$. By Neisendorfer's theorem, stated in detail in $\S 1$, any element of $\operatorname{Aut}\left(W_{p}\right)$ is induced by a (unique) element in $\operatorname{Aut}\left(T_{p}\right)$. But the image of $\left[T_{p}, T_{p}\right]$ in $\left[\left(W_{p}\right)_{(0)},\left(W_{p}\right)_{(0)}\right]=\mathbb{Q}_{p}$ is contained 
in $\left(\mathbb{Z}_{p}\right)^{k} \subset \mathbb{Z}_{p} \subset \mathbb{Q}_{p}$ (as the image of $[T, T]$ in $\left[W_{(0)}, W_{(0)}\right]$ is contained in $\mathbb{Z}^{k} \subset \mathbb{Z} \subset \mathbb{Q}$ ). Here, $R^{k}$ denotes the set consisting of the $k$ th powers of elements of $R$; similarly, we will write $\left(R^{*}\right)^{k}$ for the group consisting of the $k$ th powers of elements of $R^{*}$. Thus, in light of (2.2), we see that the double coset space in (2.4) maps surjectively to

$$
\mathbb{Q}^{*} \backslash(\widehat{\mathbb{Q}})^{*} / \prod\left(\mathbb{Z}_{p}^{*}\right)^{k}
$$

To show that the latter double coset space is uncountably infinite, it suffices to check (see [16], [17]) that there are infinitely many primes $p$ such that $\mathbb{Z}_{p}^{*} /\left(\mathbb{Z}_{p}^{*}\right)^{k}$ is non-trivial. But for $p$ odd,

$$
\left.\mathbb{Z}_{p}^{*} \cong \mathbb{Z}_{p} \oplus \mathbb{Z} / p-1 \quad \text { (see, e.g., }[26]\right) .
$$

Hence

$$
\mathbb{Z}_{p}^{*} /\left(\mathbb{Z}_{p}^{*}\right)^{k} \cong \mathbb{Z}_{p} / k \mathbb{Z}_{p} \oplus(\mathbb{Z} / p-1) / k(\mathbb{Z} / p-1) .
$$

Now, for any $k \geq 2$, there are infinitely many primes $p$ such that $p \equiv 1 \bmod k$ by Dirichlet's theorem (see, e.g., [26]). For such $p$, the second summand in (2.7) is non-trivial, and the proof of Theorem A is completed.

To prove the Addendum to Theorem A, we study the commutative diagram

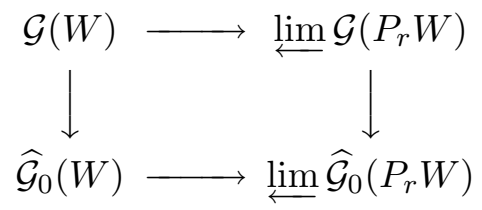

where the vertical arrows are induced by the inclusions $\mathcal{G}() \subset \widehat{\mathcal{G}}_{0}()$ and the horizontal arrows are the obvious natural maps. Since $W$, and therefore also $P_{r} W$, is a rational $H$-space, the inclusion $\mathcal{G}\left(P_{r} W\right) \subset \widehat{\mathcal{G}}_{0}\left(P_{r} W\right)$ is a bijection by a result of Belfi-Wilferson ([2; Theorem 1.1]). It follows that the right vertical arrow in (2.8) is a bijection. To prove that the left vertical arrow is also a bijection, it remains to prove that the two horizontal arrows are bijections. By [14; Lemma 6.1], the top horizontal arrow is injective provided $\operatorname{SNT}(V)$ is trivial for all $V$ in $\mathcal{G}(W)$, and is surjective provided $\operatorname{SNT}\left(W_{(p)}\right)$ is trivial for all primes $p$; the triviality of $\operatorname{SNT}(V)$ follows from the proof of Theorem $\mathrm{C}$ and the triviality of $\mathrm{SNT}\left(W_{p}\right)$ follows from Theorem C. Similarly, the bottom horizontal arrow is injective provided $\operatorname{SNT}(V)$ is trivial for all $V$ in $\widehat{\mathcal{G}}_{0}(W)$ (which again follows from the proof of Theorem $\mathrm{C}$ ) and is surjective provided $\operatorname{SNT}\left(W_{p}\right)$ is trivial for all primes $p$; the latter is a consequence of a general compactness argument - see Wilkerson ([29; Corollary II]). The proof of the Addendum to Theorem A is thereby achieved, modulo Theorem C. 
3. Proof of Theorem B. We divide the proof into two cases, as follows.

CASE 1: $k<l$. We determine $N_{0}$ and the rational homotopy structure of both $W=B\langle N\rangle$ and $W_{p}, N \geq N_{0}$, in preparation for applying (2.1).

The inclusions $S^{k} \rightarrow B$ and $S^{l} \rightarrow B$ give rise to elements $u \in \pi_{k}(B)$ and $v \in \pi_{l}(B)$. Consider the Whitehead products

$$
w_{r, s}=v \ldots v \cdot u \ldots u \cdot v,
$$

with $r$ occurrences of $v(r \geq 0)$, followed by $s$ occurrences of $u(s>0)$, followed by a single occurrence of $v$. Here we use abbreviated, bracket-free notation for Whitehead products, so that, for example,

$$
\text { v.u.u.v }=[v,[u,[u, v]]] .
$$

Note that the $w_{r, s}$ are basic products in the sense of [9] provided we require that $u<v$, which we do. Now let $P$ be any positive integer for which there are at least two distinct products $w_{r, s}, w_{\varrho, \sigma}$ having degree $P$; it is readily checked that such $P$ exist. Taking $P_{0}$ to be the least such integer, for definiteness, set

$$
N_{0}=P_{0}-(l-1)
$$

and more generally,

$$
N_{t}=N_{0}+t(l-1), \quad t \geq 0 .
$$

It is clear that the products $w_{r+t, s}, w_{\varrho+t, \sigma}$, both of degree $N_{t+1}$, are distinct for any $t \geq 0$.

By the Félix-Halperin mapping theorem (see, e.g., [6]), the rational category of $W$, cat $_{0}(W)$, equals 1 , i.e., $W$ is a rational co- $H$-space. Thus there is a rational equivalence $h_{N}$ from a one-point union of spheres $\bigvee S^{i}$ to $W$. Clearly, the induced map, $\left(h_{N}\right)_{p}$, from $\left(\bigvee S^{i}\right)_{p}$ to $W_{p}$ is also a rational equivalence. For any $N \geq N_{0}$, there is a unique $t>0$ such that $N_{t-1} \leq N<N_{t}$. Since $N_{t}$ is certainly less than or equal to $2 N$, the Hurewicz homomorphism $\pi_{N_{t}}(W) \rightarrow H_{N_{t}}(W)$ is a rational isomorphism. Thus, at least two of the spheres in the one-point union $\bigvee S^{i}$ are of dimension $N_{t}$ and $h_{N} \mid S^{N_{t}} \vee S^{N_{t}}$ may be chosen to represent the elements of $\pi_{N_{t}}(W)$ mapping to the Whitehead products $w_{r+t, s}, w_{\varrho+t, \sigma}$ via the isomorphism induced by the $N$-connective covering map $W \rightarrow B$. We denote the latter elements by $z_{r+t, s}, z_{\varrho+t, \sigma}$ and their images in $\pi_{N_{t}}^{\prime}(W)=\pi_{N_{t}}(W) /$ torsion by $z_{r+t, s}^{\prime}, z_{\varrho+t, \sigma}^{\prime}$. Write $\bigvee S^{i}=S_{1} \vee S_{2}$, where $S_{1}$ is the summand consisting of all the spheres of dimension $N_{t}$, and $S_{2}$ is the complementary summand. By a result of Bousfield and Kan ([3; Proposition VI.6.6]), the canonical map

$$
\left(S_{1}\right)_{p} \vee\left(S_{2}\right)_{p} \rightarrow\left(S_{1} \vee S_{2}\right)_{p}
$$


while not itself a homotopy equivalence, induces a map

$$
\left[\left(S_{1}\right)_{p} \vee\left(S_{2}\right)_{p}\right]_{p} \rightarrow\left(S_{1} \vee S_{2}\right)_{p}
$$

which is a homotopy equivalence. It will be convenient to regard the domains of $h_{N}$ and $\left(h_{N}\right)_{p}$ as $S_{1} \vee S_{2}$ and $\left[\left(S_{1}\right)_{p} \vee\left(S_{2}\right)_{p}\right]_{p}$, respectively.

Consider next the canonical homomorphisms

$$
\begin{aligned}
\operatorname{CAut}\left(\left(W_{(0)}\right)^{-}\right) & \rightarrow \operatorname{CAut}\left(\pi_{N_{t}}\left(\left(W_{(0)}\right)^{-}\right)\right), \\
\operatorname{Aut}\left(W_{(0)}\right) & \rightarrow \operatorname{Aut}\left(\pi_{N_{t}}\left(W_{(0)}\right)\right), \\
\operatorname{Aut}(\widehat{W}) & \rightarrow \operatorname{Aut}\left(\pi_{N_{t}}^{\prime}(\widehat{W})\right) .
\end{aligned}
$$

Denote the respective images of these homomorphisms by $I, I^{\prime}$ and $I^{\prime \prime}$. Also, denote the $p$-components of $I$, resp. $I^{\prime \prime}$, by $I(p)$, resp. $I^{\prime \prime}(p)$ (see $(2.2)$ ). The double coset space in (2.1) maps surjectively to the double coset space

$$
(f . c)_{*} I^{\prime} \backslash I / r_{*} I^{\prime \prime} .
$$

From the rational structure of $W$ and $W_{p}$ described above, we conclude that

$$
\begin{aligned}
I & =\operatorname{CAut}\left(\pi_{N_{t}}\left(\left(W_{(0)}\right)^{-}\right)\right) \cong \operatorname{Gl}(n, \widehat{\mathbb{Q}}), \\
I^{\prime} & =\operatorname{Aut}\left(\pi_{N_{t}}\left(W_{(0)}\right)\right) \cong \operatorname{Gl}(n, \mathbb{Q}),
\end{aligned}
$$

where $n \geq 2$ denotes the torsion-free rank of $\pi_{N_{t}}(W)$. The isomorphisms in (3) may be chosen to be compatible with each other, depending on the selection of an ordered basis for the free abelian group $\pi_{N_{t}}^{\prime}(W)$.

We compute $I^{\prime \prime}$ by using Neisendorfer's theorem in conjunction with (2.2), as in the proof of Theorem A. Any element $\alpha \in \operatorname{Aut}\left(W_{p}\right)$ is of the form $\beta\langle N\rangle$ for a (unique) element $\beta$ in $\operatorname{Aut}\left(B_{p}\right)$. The induced homomorphism $\beta_{\sharp}$ on homotopy groups is determined by

$$
\beta_{\sharp}(u)=a . u, \quad \beta_{\sharp}(v)=d . v,
$$

where $u, v$ are now viewed as generators of $\pi_{k}\left(B_{p}\right)=\pi_{k}(B)_{p}, \pi_{l}\left(B_{p}\right)=$ $\pi_{l}(B)_{p}$ qua $\mathbb{Z}_{p}$-modules, and $a, d \in \mathbb{Z}_{p}$. Thus,

$$
\beta_{\sharp}\left(w_{r+t, s}\right)=a^{s} \cdot b^{r+t+l} \cdot w_{r+t, s}, \quad \beta_{\sharp}\left(w_{\varrho+t, \sigma}\right)=a^{\sigma} \cdot b^{\varrho+t+1} \cdot w_{\varrho+t, \sigma},
$$

where $w_{r+t, s}, w_{\varrho+t, \sigma}$ are now viewed as elements of $\pi_{N_{t}}\left(B_{p}\right)$. Next, $z_{r+t, s}^{\prime}$, $z_{\varrho+t, \sigma}^{\prime}$ may be taken as the first two elements of an ordered basis $\mathcal{B}$ for $\pi_{N_{t}}^{\prime}(W)$; viewing $\mathcal{B}$ as an ordered basis for the free $\mathbb{Z}_{p}$-module $\pi_{N_{t}}^{\prime}\left(W_{p}\right)$, the automorphism $\alpha_{\sharp}^{\prime}$ of $\pi_{N_{t}}^{\prime}\left(W_{p}\right)$ induced by the automorphism $\alpha_{\sharp}$ of $\pi_{N_{t}}\left(W_{p}\right)$ is represented by a matrix $M=\left(m_{i j}\right)$ with respect to $\mathcal{B}$, and it follows from (3.4) that

$$
m_{21}=0
$$


For $x$ in $\mathbb{Z}_{p}$, consider the matrix

$$
M_{x}=\left(\begin{array}{cccccc}
1 & & & & & \\
x & 1 & & & & \\
& & 1 & & & \\
& & & 1 & & \\
& & & & 1 & \\
& & & & & 1
\end{array}\right) \text {, }
$$

all the non-displayed entries being 0 . Since $M_{x} \cdot M_{y}^{-1}=M_{x-y}$, it follows from (3.5) that for $x \neq y$, the matrices $M_{x}, M_{y}$ determine distinct elements in the coset space $I(p) / r_{*} I^{\prime \prime}(p)$. Therefore, this coset space, and hence also the coset space in (3.1), is uncountably infinite. This completes the proof of Case 1 of Theorem B.

CASE 2: $k=l$. We continue with the notation used in Case 1 and consider the three distinct Whitehead products $w_{0,3}, w_{1,2}, w_{2,1}$, each of degree $4 k-3$. As in Case 1 , we set

$$
N_{0}=3 k-2,
$$

more generally,

$$
N_{t}=N_{0}+t(k-1), \quad t \geq 0,
$$

and for $N_{t-1} \leq N<N_{t}, t>0$, find a rational equivalence $h_{N}$ from a onepoint union of spheres $\bigvee S^{i}$ to $W$. In Case 2, at least three of the spheres in the one-point union $\bigvee S^{i}$ are of dimension $N_{t}$ and $h_{N} \mid S^{N_{t}} \vee S^{N_{t}} \vee S^{N_{t}}$ may be chosen to represent the elements $z_{0,2+t}, z_{1,1+t}, z_{1+t, 1}$ in $\pi_{N_{t}}(W)$ mapping to $w_{0,2+t}, w_{1,1+t}, w_{1+t, 1}$. The isomorphisms of $(3)$ in Case 1 hold also for Case 2 except that now $n \geq 3$, but the analog of (3.3) for Case 2 becomes

$$
\beta_{\sharp}(u)=a . u+b . v, \quad \beta_{\sharp}(v)=c . u+d . v,
$$

where $a, b, c, d \in \mathbb{Z}_{p}$. The elements $z_{0,2+t}^{\prime}, z_{1,1+t}^{\prime}, z_{1+t, 1}^{\prime} \in \pi_{N_{t}}^{\prime}(W)$ may be taken as the first, second and last elements of an ordered basis $\mathcal{B}$ for $\pi_{N_{t}}^{\prime}(W)$. Viewing $\mathcal{B}$ as an ordered basis of the free $\mathbb{Z}_{p}$-module $\pi_{N_{t}}^{\prime}\left(W_{p}\right)$, we study the matrix $M=\left(m_{i j}\right)$ representing the automorphism $\alpha_{\sharp}^{\prime}$ of $\pi_{N_{t}}^{\prime}\left(W_{p}\right)$ with respect to $\mathcal{B}$. From (3.6), computation shows that

$$
m_{11}=\Delta \cdot a^{2+t}, \quad m_{n 1}=\Delta \cdot c^{2+t}, \quad m_{21}=\Delta \cdot a^{1+t} \cdot c,
$$

where $\Delta=a . d+(-1)^{k} b . c$. Note that for $k$ odd, $\Delta=\delta$, the determinant of the automorphism $\beta_{\sharp}$ of $\pi_{k}\left(B_{p}\right)$, hence is in $\mathbb{Z}_{p}^{*}$. We claim that, as in Case 1 , the map sending $x$ in $\mathbb{Z}_{p}$ to the coset of $M_{x}$ in $I(p) / r_{*} I^{\prime \prime}(p)$ is injective. 
Indeed, if $M_{x} \cdot M_{y}^{-1} \in r_{*} I^{\prime \prime}(p)$, then (3.7) implies

$$
\begin{aligned}
& 1=\Delta \cdot a^{2+t}, \\
& 0=\Delta \cdot c^{2+t}, \quad \text { hence } c=0, \\
& x-y=a^{1+t} \cdot c, \quad \text { hence } \quad x=y .
\end{aligned}
$$

This completes the proof of Case 2 of Theorem B.

We conclude this section with some remarks regarding the proof of Case 2 of Theorem B. If we fix ordered bases for $\operatorname{Aut}\left(B_{p}\right)=\operatorname{Aut}\left(\pi_{k}\left(B_{p}\right)\right)$ and $\operatorname{Aut}\left(\pi_{N_{t}}^{\prime}\left(W_{p}\right)\right)=\operatorname{Aut}\left(\pi_{N_{t}}^{\prime}\left(B_{p}\right)\right)$, the homomorphism

$$
\operatorname{Aut}\left(\pi_{k}\left(B_{p}\right)\right) \rightarrow \operatorname{Aut}\left(\pi_{N_{t}}^{\prime}\left(W_{p}\right)\right)
$$

implicit in the proof of Case 2 of Theorem $\mathrm{B}$, is represented by a homomorphism

$$
f: \operatorname{Gl}\left(2, \mathbb{Z}_{p}\right) \rightarrow \operatorname{Gl}\left(n, \mathbb{Z}_{p}\right), \quad n>2 .
$$

Our explicit computations lead to the conclusion that the coset space $\operatorname{Gl}\left(n, \mathbb{Z}_{p}\right) / f\left(\mathrm{Gl}\left(2, \mathbb{Z}_{p}\right)\right)$ is uncountably infinite. Also, in the proof of Theorem $\mathrm{D}$ below, a similar homomorphism

$$
f: \operatorname{Gl}(2, \mathbb{Z}) \rightarrow \operatorname{Gl}(n, \mathbb{Z}), \quad n>2,
$$

appears implicitly, with the property that the coset space $\mathrm{Gl}(n, \mathbb{Z}) / f(\mathrm{Gl}(2, \mathbb{Z}))$ is (countably) infinite. The question raised near the end of the introduction asks whether the conclusions about the size of the coset spaces are valid for general homomorphisms $f$.

Here are three examples of the foregoing. In contrast with the first example, the latter two are not strict illustrations of the recipe used in the proof of Case 2 of Theorem B, but are rather variations of that recipe. In all three examples, we fix the ordered basis $\{u, v\}$ for $\pi_{k}\left(B_{p}\right)$.

EXAmple 1 . Let $k=3$, so that $N_{0}=7$, and set $N=7$, so that $N_{1}=9$. Fixing the ordered basis $\left\{z_{0,3}^{\prime}, z_{1,2}^{\prime}, z_{2,1}^{\prime}\right\}$ for $\pi_{9}^{\prime}\left(W_{p}\right)$, we compute

$$
f\left(\left(\begin{array}{ll}
a & b \\
c & d
\end{array}\right)\right)=M=\delta .\left(\begin{array}{ccc}
a^{2} & 2 a b & b^{2} \\
a c & a d+b c & b d \\
c^{2} & 2 c d & d^{2}
\end{array}\right),
$$

whose determinant is $\delta^{6}$.

EXAmple 2. Let $k=3$ again, but now set $N=3$. Fixing the ordered basis $\left\{z_{0,2}^{\prime}, z_{1,1}^{\prime}\right\}$ for $\pi_{7}^{\prime}\left(W_{p}\right)$, we consider the homomorphism

$$
\operatorname{Aut}\left(\pi_{3}\left(B_{p}\right)\right) \rightarrow \operatorname{Aut}\left(\pi_{7}^{\prime}\left(W_{p}\right)\right)
$$

and compute

$$
f\left(\left(\begin{array}{ll}
a & b \\
c & d
\end{array}\right)\right)=\delta \cdot\left(\begin{array}{ll}
a & b \\
c & d
\end{array}\right)
$$


whose determinant is $\delta^{2}$. In this example, the coset space $\operatorname{Gl}\left(2, \mathbb{Z}_{p}\right) / f\left(\operatorname{Gl}\left(2, \mathbb{Z}_{p}\right)\right)$ is non-trivial since the determinant homomorphism induces a surjection

$$
\mathrm{Gl}\left(2, \mathbb{Z}_{p}\right) / f\left(\mathrm{Gl}\left(2, \mathbb{Z}_{p}\right)\right) \rightarrow \mathbb{Z}_{p}^{*} /\left(\mathbb{Z}_{p}^{*}\right)^{2}
$$

The method of proof of Theorem A then shows that $\widehat{\mathcal{G}}_{0}(W)$ is uncountably infinite.

ExAmple 3 . Let $k=4$, so that $N_{0}=10$, but set $N=4$. Denote by $(u u)^{\prime},(u v)^{\prime},(v v)^{\prime}$ the canonical images in $\pi_{7}^{\prime}\left(B_{p}\right)$ of the Whitehead products $u u, u v, v v$. (Of course, $u u$ and $v v$ are not basic products.) Note that $\left\{(u u)^{\prime},(u v)^{\prime},(v v)^{\prime}\right\}$ is an ordered basis for $\pi_{7}^{\prime}\left(B_{p}\right)$, at least for $p$ odd. Fixing this basis, we consider the homomorphism

$$
\operatorname{Aut}\left(\pi_{4}\left(B_{p}\right)\right) \rightarrow \operatorname{Aut}\left(\pi_{7}^{\prime}\left(B_{p}\right)\right)
$$

and compute

$$
f\left(\left(\begin{array}{ll}
a & b \\
c & d
\end{array}\right)\right)=\left(\begin{array}{ccc}
a^{2} & 2 a b & b^{2} \\
a c & a d+b c & b d \\
c^{2} & 2 c d & d^{2}
\end{array}\right)
$$

whose determinant is $\delta^{3}$.

The fact that in all three examples, the determinant of $f\left(\left(\begin{array}{ll}a & b \\ c & d\end{array}\right)\right)$ is of the form $\delta^{e}, e>1$, is no accident. It can be shown that in the context of the proof of Case 2 of Theorem B, the determinant of $f\left(\left(\begin{array}{ll}a & b \\ c & d\end{array}\right)\right)$ is always of this form.

4. Proof of Theorem C. First suppose $m<2 n$. Then, reverting to the notation of $\S 2, W=T\langle m\rangle=T$, a finite CW-complex, and $W_{(p)}=$ $T\langle m\rangle_{(p)}=T_{(p)}$, a finite-dimensional $\mathrm{CW}$-complex. It is thus clear that the conclusion of Theorem $\mathrm{C}$ holds in this case.

Next suppose $m>2 n k-2$. Then all the homotopy groups of $W$ and $W_{(p)}$ are finite. The conclusion of Theorem $\mathrm{C}$ follows in this case from [29; Corollary II].

For the remainder of this section, we focus on the interesting range $2 n \leq$ $m \leq 2 n k-2$, and utilize the following criterion of McGibbon and Møller ([14; Theorem 3]): If $Z$ is the $P$-localization of a 1-connected, finite type, rational $H$-space, where $P$ is a collection of primes, then $\operatorname{SNT}(Z)$ is trivial if and only if the image of the canonical (anti)homomorphism from $\operatorname{Aut}(Z)$ to $\operatorname{Aut}\left(H \leq \mu\left(Z ; \mathbb{Z}_{(P)}\right)\right)$ is of finite index for all $\mu$. By the latter automorphism group, we mean the group of graded ring automorphisms of the graded ring obtained from $H^{*}\left(Z ; \mathbb{Z}_{(P)}\right)$ by replacing the cohomology groups in degrees $>\mu$ by 0 . This criterion applies to the situation in Theorem $\mathrm{C}$ since $W$ and $W_{(p)}$ are 1-connected, finite type, rational $H$-spaces. 
Since $H^{i}(W ; \mathbb{Z})$ is finite if $i \neq 2 n k-1$, and $H^{2 n k-1}(W ; \mathbb{Z})$ is finitely generated abelian with torsion-free rank $1, \operatorname{Aut}\left(H^{\leq \mu}(W ; \mathbb{Z})\right)$ is itself finite for all $\mu$ and the criterion for triviality of $\operatorname{SNT}(W)$ is satisfied, a fortiori. It then remains to show that the image of the canonical (anti)homomorphism from $\operatorname{Aut}\left(W_{(p)}\right)$ to $\operatorname{Aut}\left(H^{2 n k-1}\left(W_{(p)} ; \mathbb{Z}_{(p)}\right) /\right.$ torsion $)=\mathbb{Z}_{(p)}^{*}$ is of finite index. Equivalently, it suffices to show that the image of the canonical homomorphism from $\operatorname{Aut}\left(W_{(p)}\right)$ to $\operatorname{Aut}\left(\pi_{2 n k-1}^{\prime}\left(W_{(p)}\right)\right) \cong \operatorname{Aut}\left(H_{2 n k-1}\left(W_{(p)} ; \mathbb{Z}\right) /\right.$ torsion) is of finite index, since these last two automorphism groups are (anti)isomorphic to $\operatorname{Aut}\left(H^{2 n k-1}\left(W_{(p)} ; \mathbb{Z}_{(p)}\right) /\right.$ torsion). Our strategy will be to show that the image of the canonical map from $\operatorname{Aut}\left(W_{p}\right)$ to $\operatorname{Aut}\left(\pi_{2 n k-1}^{\prime}\left(W_{p}\right)\right)$ is sufficiently large in an appropriate sense, and then to pass from $W_{p}$ to $W_{(p)}$ using a local arithmetic square argument. The details follow.

Lemma 4.1. There exists a positive integer e, depending only on $T$, with the following property: If $d$ is any integer, there exists an $\alpha_{d}$ in $[T, T]$ such that

$$
\left(\alpha_{d}\right)_{\sharp}: \pi_{2 n}(T) \rightarrow \pi_{2 n}(T) \text { is multiplication by } d^{e},
$$

and consequently,

$$
\left(\alpha_{d}\right)_{\sharp}: \pi_{2 n k-1}^{\prime}(T) \rightarrow \pi_{2 n k-1}^{\prime}(T) \text { is multiplication by } d^{E} \text {, where } E=k e \text {. }
$$

Proof. The result is clear, with $e=1$, in case (i) and therefore also in case (ii) (even if $k=\infty$ ). For case (iv), a theorem of Sullivan ([27; pp. 58-59, Remark IV]) asserts that (4.1) holds for $d$ odd, with $e=2$ (even if $k=\infty$ ), and a theorem of McGibbon ([13; Proposition 2.4]) asserts that (4.1) holds for $d$ even. (A classical homotopy theory calculation shows that, already for $\mathbb{H} P^{2}$, an $\alpha_{d}$ satisfying (4.1) exists precisely when

$$
d^{e}\left(d^{e}-1\right) \equiv 0 \bmod 24 .
$$

Hence, for $d=2$, we see that $e \geq 4$.) Finally, for case (v), an argument similar to the one referred to in the previous sentences shows an $\alpha_{d}$ satisfying (4.1) exists precisely when

$$
d^{e}\left(d^{e}-1\right) \equiv 0 \bmod 240 .
$$

But this congruence holds for $d=2,3$ or 5 , with $e=4$, and for $d$ relatively prime to 240 , with $e=64$, by the Euler-Fermat theorem.

Lemma 4.2. For any $x$ in $\mathbb{Z}_{p}$, there exists a $\beta_{x}$ in $\left[T_{p}, T_{p}\right]$ such that

$$
\left(\beta_{x}\right)_{\sharp}: \pi_{2 n}\left(T_{p}\right) \rightarrow \pi_{2 n}\left(T_{p}\right) \text { is multiplication by } x^{e} \text {, }
$$

and consequently

$$
\left(\beta_{x}\right)_{\sharp}: \pi_{2 n k-1}^{\prime}\left(T_{p}\right) \rightarrow \pi_{2 n k-1}^{\prime}\left(T_{p}\right) \text { is multiplication by } x^{E} \text {. }
$$

Moreover, if $x$ is in $\mathbb{Z}_{p}^{*}$, then any such $\beta_{x}$ actually lies in $\operatorname{Aut}\left(T_{p}\right)$. 
Proof. Let $\left(d_{i}\right)$ be a sequence of integers converging to $x$ in the $p$-adic topology. For each $d_{i}$, let $\alpha_{d_{i}}$ be as in Lemma 4.1 and let

$$
\beta_{d_{i}}=\left(\alpha_{d_{i}}\right)_{p}
$$

the $p$-completion of $\alpha_{d_{i}}$. The homotopy set $\left[T_{p}, T_{p}\right]$ has a natural compact, Hausdorff topology $([27])$ and so the sequence $\left(\beta_{d_{i}}\right)$ admits a convergent subsequence. If $\beta_{x}$ denotes the limit of such a subsequence, then (4.3) is readily verified for this choice of $\beta_{x}$.

Suppose now that $x$ is in $\mathbb{Z}_{p}^{*}$. We will show that $\beta_{x}$ induces automorphisms on all homotopy groups, hence lies in $\operatorname{Aut}\left(T_{p}\right)$. First, from (4.3), $\beta_{x}$ induces an automorphism on $H_{2 n}\left(T_{p}\right)$, hence, a fortiori, on $H_{2 n}\left(T_{p} ; \mathbb{Z} / p^{r}\right)$ (and $\left.H^{2 n}\left(T_{p} ; \mathbb{Z} / p^{r}\right)\right), r>0$. From the homological structure of $T_{p}$ with coefficients in $\mathbb{Z}_{p^{r}}$, we see that $\beta_{x}$ induces an automorphism on $H_{j}\left(T_{p} ; \mathbb{Z} / p^{r}\right)$ for all $j \geq 0$ and all $r>0$. It then follows from [21; Corollary 3.10] that $\beta_{x}$ induces an automorphism on $\pi_{j}\left(T_{p} ; \mathbb{Z} / p^{r}\right)$, the homotopy groups with coefficients in $\mathbb{Z} / p^{r}$, for all $j \geq 0$ and all $r>0$. Finally, using the functorial short exact sequence (universal coefficient theorem; see, e.g., [21; Proposition 1.4])

$$
0 \rightarrow \pi_{j}\left(T_{p}\right) \otimes \mathbb{Z} / p^{r} \rightarrow \pi_{j}\left(T_{p} ; \mathbb{Z} / p^{r}\right) \rightarrow \operatorname{Tor}\left(\pi_{j-1}\left(T_{p}\right), \mathbb{Z} / p^{r}\right) \rightarrow 0,
$$

in conjunction with $(4.3),(4.4)$ and the fact that $\pi_{j}^{\prime}\left(T_{p}\right)=0$ for all $j \neq 2 n$, $2 n k-1$, we conclude that $\beta_{x}$ induces automorphisms on $\pi_{j}\left(T_{p}\right)$ for all $j \geq 0$, as desired.

Next, let $\eta \in \mathbb{Z}_{(p)}^{*}$ be such that

$$
C(\eta)=x^{E} \quad \text { for some } x \in \mathbb{Z}_{p}^{*}
$$

where $C: \mathbb{Z}_{(p)} \subset \mathbb{Z}_{p}$ is the $p$-completion homomorphism, and let $\beta=\beta_{x} \in$ $\operatorname{Aut}\left(T_{p}\right)$ be as in Lemma 4.2. Clearly, $\beta\langle m\rangle \in \operatorname{Aut}\left(W_{p}\right)$ and, by (4.4),

$$
(\beta\langle m\rangle)_{\sharp}: \pi_{2 n k-1}^{\prime}\left(W_{p}\right) \rightarrow \pi_{2 n k-1}^{\prime}\left(W_{p}\right) \text { is multiplication by } x^{E} \text {. }
$$

We also have $\gamma \in \operatorname{Aut}\left(W_{(0)}\right)$ (uniquely) defined by the condition that

$$
\gamma_{\sharp}: \pi_{2 n k-1}\left(W_{(0)}\right) \rightarrow \pi_{2 n k-1}\left(W_{(0)}\right) \text { is multiplication by } R(\eta),
$$

where $R: \mathbb{Z}_{(p)}^{*} \subset \mathbb{Q}^{*}$ is the rationalization homomorphism. From the homotopy-pullback diagram

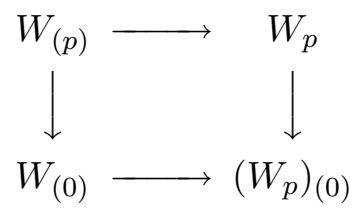

(local arithmetic square), we readily infer the existence of an $\epsilon$ in $\left[W_{(p)}, W_{(p)}\right]$ whose images in $\operatorname{Aut}\left(W_{p}\right)$ and $\operatorname{Aut}\left(W_{(0)}\right)$ are, respectively, $\beta\langle m\rangle$ and $\gamma$. By a homotopical Mayer-Vietoris argument, we see that $\epsilon$ is in $\operatorname{Aut}\left(W_{(p)}\right)$ and 
that

$$
\epsilon_{\sharp}: \pi_{2 n k-1}^{\prime}\left(W_{(p)}\right) \rightarrow \pi_{2 n k-1}^{\prime}\left(W_{(p)}\right) \text { is multiplication by } \eta .
$$

We have thus shown that the canonical homomorphism from $\operatorname{Aut}\left(W_{(p)}\right)$ to $\operatorname{Aut}\left(\pi_{2 n k-1}^{\prime}\left(W_{(p)}\right)\right)$ contains (in fact equals) $C^{-1}\left(\left(\mathbb{Z}_{p}^{*}\right)^{E}\right)$. But since $\left(\mathbb{Z}_{p}^{*}\right)^{E}$ has finite index in $\mathbb{Z}_{p}^{*}$ by $(2.6), C^{-1}\left(\left(\mathbb{Z}_{p}^{*}\right)^{E}\right)$ has finite index in $\mathbb{Z}_{(p)}^{*}$. This completes the proof of Theorem C.

REMark. While $\left(\mathbb{Z}_{p}^{*}\right)^{E}$ has finite index in $\mathbb{Z}_{p}^{*}$, it is not true that $\left(\mathbb{Z}_{(p)}^{*}\right)^{E}$ has finite index in $\mathbb{Z}_{(p)}^{*}$ if $E>1$. In fact, $\mathbb{Z}_{(p)}^{*} /\left(\mathbb{Z}_{(p)}^{*}\right)^{E}$ is isomorphic to a direct sum of countably many copies of $\mathbb{Z} / E$; generators are provided by the cosets determined by the primes $q \neq p$. As a consequence, there is no analog of Neisendorfer's theorem for $p$-localization; that is, not every element of $\left[W_{(p)}, W_{(p)}\right]$ "comes from" an element of $\left[T_{(p)}, T_{(p)}\right]$.

5. Proof of Theorem D. We now revert to the notation of $\S 3$, writing $W$ for the $N$-connective covering $B\langle N\rangle=\left(S^{k} \vee S^{l}\right)\langle N\rangle$. In order to prove Theorem D, we will utilize a criterion of McGibbon and Møller ([15; Theorem 1]) dual to the criterion used in $\S 4$, namely: If $Z$ is a 1 -connected, finite type, rational co- $H$-space, then $\operatorname{SNT}(Z)$ is trivial if and only if the image of the canonical homomorphism from $\operatorname{Aut}(Z)$ to $\operatorname{Aut}\left(\pi_{\leq \mu}(Z)\right)$ is of finite index for all $\mu$. By the latter automorphism group, we mean the group of automorphisms of the graded Lie ring obtained from $\pi_{*}(Z)$ by replacing the homotopy groups in degrees $>\mu$ by 0 . This criterion applies to the situation in Theorem $\mathrm{D}$ since $W$ is, as noted in $\S 3$, a 1-connected, finite type, rational co- $H$-space.

We will prove Theorem $D$ by showing that the image of the canonical homomorphism from $\operatorname{Aut}(W)$ to $\operatorname{Aut}\left(\pi_{\leq N_{t}}(W)\right)$ is of infinite index. Since $N_{t}$ is certainly less than or equal to $2 N-2$, the Lie ring structure on $\pi_{\leq N_{t}}(W)$ is trivial and it therefore suffices to show that the image of the canonical homomorphism from $\operatorname{Aut}(W)$ to $\operatorname{Aut}\left(\pi_{N_{t}}(W)\right)$, or from $\operatorname{Aut}(W)$ to $\operatorname{Aut}\left(\pi_{N_{t}}^{\prime}(W)\right)$, is of infinite index. To that end, pick a prime $p$ arbitrarily and consider the commutative square

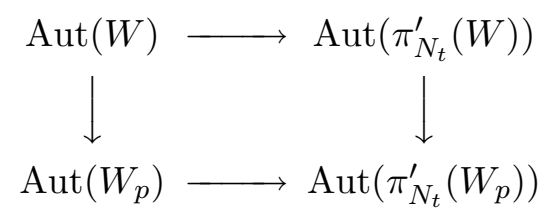

with vertical arrows induced by $p$-completion $W \rightarrow W_{p}$. With respect to the ordered bases $\mathcal{B}$ for $\pi_{N_{t}}^{\prime}(W)$ (or for $\pi_{N_{t}}^{\prime}\left(W_{p}\right)$ ) described in $\S 3$, Aut $\left(\pi_{N_{t}}^{\prime}(W)\right.$ ) and $\operatorname{Aut}\left(\pi_{N_{t}}^{\prime}\left(W_{p}\right)\right)$ may be identified with $\operatorname{Gl}(n, \mathbb{Z})$ and $\operatorname{Gl}\left(n, \mathbb{Z}_{p}\right)$, respectively, and the right vertical arrow may be identified with the homomorphism 
from $\operatorname{Gl}(n, \mathbb{Z})$ to $\operatorname{Gl}\left(n, \mathbb{Z}_{p}\right)$ induced by $p$-completion $\mathbb{Z} \subset \mathbb{Z}_{p}$. It follows that an element in the image of the top horizontal arrow in (5.1) has matrix representative $M$ satisfying the properties derived in $\S 3((3.5),(3.7))$ except that the entries of $M$ are in $\mathbb{Z}$. The computations of $\S 3$ (see especially (3.8) in Case 2: $k=l$ ) then show that the matrices $M_{x}$ of $\S 3, x \in \mathbb{Z}$, determine mutually distinct cosets modulo the image of $\operatorname{Aut}(W)$ in $\operatorname{Gl}(n, \mathbb{Z})$. This completes the proof of Theorem D.

Appendix 1. We describe here a family of spaces with each $X$ in the family having $\mathcal{G}(X)$ at most countably infinite and $\widehat{\mathcal{G}}_{0}(X)$ uncountably infinite. We begin with the spaces $\operatorname{BSU}(3)$, the classifying space of the special unitary group $\mathrm{SU}(3)$, and $K=K(\mathbb{Z}, 4) \times K(\mathbb{Z}, 6)$. Observe that these spaces are rationally equivalent and write $\mathcal{R}$ for a common rationalization. Let $P$ be a finite set of primes, each $>3$, let $Q$ be the set of all primes not in $P$, and choose rationalization maps

$$
\operatorname{BSU}(3)_{(P)} \rightarrow \mathcal{R}, \quad K_{(Q)} \rightarrow \mathcal{R},
$$

which we may assume to be fibrations. We then define $X$ to be the pullback of these two maps. Thus $X$ is a "Zabrodsky mix" of BSU(3) and $K$; that is,

$$
X_{(P)} \simeq \mathrm{BSU}(3)_{(P)}, \quad X_{(Q)} \simeq K_{(Q)} .
$$

According to [20; Theorem 2.1], if $P$ contains at least 2 primes, there are, up to homotopy, exactly countably (infinitely) many nilpotent, $P$-local spaces of finite type over $\mathbb{Z}_{(P)},\left\{U_{1}, U_{2}, \ldots\right\}$, satisfying

$$
\left(U_{i}\right)_{(p)} \simeq \operatorname{BSU}(3)_{(p)}, \quad i \geq 1, j \in P .
$$

Of course, if $P$ is the singleton set $\{p\}$, there is only one $U_{i}$ as in (A1.2), namely $\operatorname{BSU}(3)_{(p)}$ itself. Furthermore, up to homotopy, the only nilpotent $Q$-local space of finite type over $\mathbb{Z}_{(Q)}$ whose $p$-localization is homotopy equivalent to $K_{(p)}$, for all $p \in Q$, is $K_{(Q)}$ itself.

Suppose now that $Y$ (more accurately, the homotopy type of $Y$ ) is in $\mathcal{G}(X)$. Then

$$
Y_{(P)} \simeq U_{i_{0}} \quad \text { for some } i_{0},
$$

by (A1.1) and (A1.2). Similarly,

$$
Y_{(Q)} \simeq K_{(Q)}
$$

By [10; II, Theorem 5.9], $Y$ is homotopy equivalent to the pullback of the rationalization maps

$$
R_{(P)}: Y_{(P)} \rightarrow Y_{(0)}, \quad R_{(Q)}: Y_{(Q)} \rightarrow Y_{(0)}
$$

induced by the rationalization map $R: Y \rightarrow Y_{(0)}$ (assuming $R_{(P)}$ and $R_{(Q)}$ to be fibrations). It follows from this, together with (A1.3) and (A1.4), that $Y$ 
is homotopy equivalent to the pullback of appropriate rationalization maps

$$
r_{(P)}: U_{i_{0}} \rightarrow Y_{(0)}, \quad r_{(Q)}: K_{(Q)} \rightarrow Y_{(0)}
$$

(once again assuming $r_{(P)}$ and $r_{(Q)}$ are fibrations). In particular, the homotopy type of $Y$ is completely determined by the homotopy classes of $r_{(P)}$ and $r_{(Q)}$. But the number of choices for the homotopy classes of $r_{(P)}$ and $r_{(Q)}$ is countably infinite since the full homotopy sets

$$
\begin{aligned}
& {\left[U_{i_{0}}, Y_{(0)}\right] \cong H^{4}\left(U_{i_{0}} ; \mathbb{Q}\right) \times H^{6}\left(U_{i_{0}} ; \mathbb{Q}\right),} \\
& {\left[K_{Q}, Y_{(0)}\right] \cong H^{4}\left(K_{(Q)} ; \mathbb{Q}\right) \times H^{6}\left(K_{(Q)} ; \mathbb{Q}\right)}
\end{aligned}
$$

are clearly countably infinite. Thus $\mathcal{G}(X)$ is at most countably infinite, and exactly countably infinite when $P$ contains at least two primes.

To see that $\widehat{\mathcal{G}_{0}}(X)$ is uncountably infinite, we use the double coset formula (2.1), which in the present situation reduces to

$$
\mathbb{Q}^{*} \times \mathbb{Q}^{*} \backslash(\widehat{\mathbb{Q}})^{*} \times(\widehat{\mathbb{Q}})^{*} / r_{*} \operatorname{Aut}(\widehat{X}) .
$$

By examining the computation in [14; Ex. H], we find that the $p$-component of $r_{*} \operatorname{Aut}(\widehat{X})$ is

$$
J(p)=\left\{\left(x^{2}, x^{3}\right) \mid x \in \mathbb{Z}_{p}^{*}\right\}, \quad \text { provided } p \in P .
$$

(For $p \in Q$, the $p$-component of $r_{*} \operatorname{Aut}(\widehat{X})$ is all of $\mathbb{Z}_{p}^{*} \times \mathbb{Z}_{p}^{*}$.)

We next observe that the quotient $\mathbb{Z}_{p}^{*} \times \mathbb{Z}_{p}^{*} / J(p)$ is uncountably infinite if $p \in P$. In fact, by (2.6), this quotient contains a summand isomorphic to $\mathbb{Z}_{p} \oplus \mathbb{Z}_{p} /\left\{(2 x, 3 x) \mid x \in \mathbb{Z}_{p}\right\}$, which is itself isomorphic to $\mathbb{Z}_{p}$. It follows that the double coset in $(\mathrm{A} 1.5)$, and hence $\widehat{\mathcal{G}}_{0}(X)$, is uncountably infinite.

Appendix 2. This appendix consists largely of speculative remarks, which we hope to develop on a future occasion. However, we do include the following partial answer to the Question raised in $\S 1$, whose formulation and proof owe much to suggestions of Pierre de la Harpe.

Theorem E. Let $f: \operatorname{Gl}(\nu, \mathbb{Z}) \rightarrow \operatorname{Gl}(n, \mathbb{Z})$ be a homomorphism, $\nu<n$. If $\operatorname{ker}(f)$ is finite, then the coset space $\operatorname{Gl}(n, \mathbb{Z}) / f(\mathrm{Gl}(\nu, \mathbb{Z}))$ is infinite.

We point out that the condition that $\operatorname{ker}(f)$ be finite is satisfied in the situation considered in $\S 5$, as can be verified by making matrix computations similar to those carried out in $\S 3$.

Proof of Theorem E. Our argument relies on the formula

$$
\operatorname{vcd}(\operatorname{Sl}(k, \mathbb{Z}))=\frac{k(k-1)}{2},
$$

where $\operatorname{vcd}(G)$ stands for the "virtual cohomological dimension" of the group $G$, that is, the cohomological dimension, $\operatorname{cd}(H)$, of any torsion-free subgroup $H$ of finite index in $G$ (provided such subgroups exist); see [25] 
or [4] for a discussion of vcd, and [4] for a proof of (A2.1). Since $\operatorname{Sl}(k, \mathbb{Z})$ is of index 2 in $\operatorname{Gl}(k, \mathbb{Z}),(\mathrm{A} 2.1)$ is also valid for $\operatorname{Gl}(k, \mathbb{Z})$.

Let then $H$ be a torsion-free subgroup of finite index in $\operatorname{Gl}(\nu, \mathbb{Z})$. By (A2.1), we have

$$
\operatorname{cd}(H)=\frac{\nu(\nu-1)}{2}
$$

Assume, for a contradiction, that $\mathrm{Gl}(n, \mathbb{Z}) / f(\mathrm{Gl}(\nu, \mathbb{Z}))$ is finite, and consider the homomorphism $\phi$ from $H$ to $f(H)$ induced by $f$. Since $\operatorname{ker}(f)$ is finite and $H$ is torsion-free, $\phi$ is an isomorphism, so that

$$
\operatorname{cd}(f(H))=\frac{\nu(\nu-1)}{2} .
$$

But $f(H)$ is of finite index in $f(\operatorname{Gl}(\nu, \mathbb{Z}))$, which is, by assumption, of finite index in $\operatorname{Gl}(n, \mathbb{Z})$. Hence, again by $(\mathrm{A} 2.1)$,

$$
\operatorname{cd}(f(H))=\frac{n(n-1)}{2},
$$

and we have arrived at our contradiction.

In the case $\nu=2$, there are alternative approaches to proving Theorem E based on [1], [7] or [18] rather than (A2.1).

If the assumption that $\operatorname{ker}(f)$ be finite is dropped, then the technique of proof of Theorem E fails. As de la Harpe points out, there is a substantial difference between the case $\nu>2$, where the conclusion of Theorem $\mathrm{E}$ is probably true, and the case $\nu=2$, where the conclusion of Theorem $\mathrm{E}$ is probably false. We will discuss only the case $\nu=2$ here. In that case, there is an example of Conder (implicit in [5]) of a homomorphism from $\mathrm{Sl}(2, \mathbb{Z})$ (actually from $\operatorname{PSl}(2, \mathbb{Z})$ ) to $\mathrm{Sl}(3, \mathbb{Z})$ such that $\mathrm{Sl}(3, \mathbb{Z}) / f(\mathrm{Sl}(2, \mathbb{Z})$ ) is finite, and examples of Tamburini, Wilson and Gavioli ([28]) of epimorphisms from $\operatorname{Sl}(2, \mathbb{Z})$ to $\operatorname{Sl}(n, \mathbb{Z}), n \geq 28$; see also [8; III.39] for further discussion and references. It therefore seems plausible that there should exist homomorphisms from $\operatorname{Gl}(2, \mathbb{Z})$ to $\operatorname{Gl}(n, \mathbb{Z})$, for various $n>2$, with $\operatorname{Gl}(n, \mathbb{Z}) / f(\mathrm{Gl}(2, \mathbb{Z}))$ finite, although Marston Conder has pointed out to us that the particular homomorphism he constructs in [5] does not extend to a homomorphism from $\operatorname{Gl}(2, \mathbb{Z})$ to $\operatorname{Gl}(n, \mathbb{Z})$.

The examples in [5] and [28] are presumably more "exotic" than the homomorphisms which arise in $\S 3$ and $\S 5$. These latter homomorphisms are of a rather specialized sort; for any such $f$, and any $\left(\begin{array}{ll}a & b \\ c & d\end{array}\right)$ in $\operatorname{Gl}(2, A)$, $A=\mathbb{Z}$ or $\mathbb{Z}_{p}$, computation shows that the entries of $f\left(\left(\begin{array}{ll}a & b \\ c & d\end{array}\right)\right)$ are polynomial functions, with coefficients in $\mathbb{Z}$, of $a, b, c$ and $d$. For such $f$, one might expect to be able to approach the Question using the theory of algebraic groups. However, we have been warned by experts (Moskowitz, Hoobler) to tread carefully since: (1) the coefficient rings $\mathbb{Z}, \mathbb{Z}_{p}$ are not fields; (2) the 
traditional coset spaces are not the natural ones arising in the theory of algebraic groups.

Finally, we make a couple of remarks about the Question when the coefficient ring is $\mathbb{Z}_{p}$. First, there is a version of (A2.1) in that case. Indeed, according to Henn, classical work of Lazard ([12]) may be used to prove the formula

$$
\operatorname{vcd}\left(\operatorname{Sl}\left(k, \mathbb{Z}_{p}\right)\right)=k(k-1),
$$

provided we work with "continuous cohomology". However, $\operatorname{since} \operatorname{Sl}\left(k, \mathbb{Z}_{p}\right)$ does not have finite index in $\operatorname{Gl}\left(k, \mathbb{Z}_{p}\right)$, it is not clear how to use (A2.3) to derive a version of Theorem $\mathrm{E}$ in the case of $\mathbb{Z}$ replaced by $\mathbb{Z}_{p}$. Secondly, we wonder whether there is a "differentiable" approach to the Question. To explain, first note that for any differentiable (i.e., $C^{\infty}$ ) homomorphism $f: \operatorname{Gl}(\nu, \mathbb{R}) \rightarrow \operatorname{Gl}(n, \mathbb{R}), \nu<n, \mathbb{R}$ the reals, $f(\operatorname{Gl}(\nu, \mathbb{R}))$ has Lebesgue measure zero in $\operatorname{Gl}(n, \mathbb{R})$, by Sard's lemma (see, e.g., [19]), from which it easily follows that $\operatorname{Gl}(n, \mathbb{R}) / f(\mathrm{Gl}(\nu, \mathbb{R}))$ is uncountably infinite. Now one can also do analysis over $\mathbb{Q}_{p}$, and $\mathbb{Z}_{p}$ is an open, dense subspace of $\mathbb{Q}_{p}$. We ask: Is there a $p$-adic version of Sard's lemma? If so, can we thereby deduce an affirmative answer to the Question in case $f: \operatorname{Gl}\left(\nu, \mathbb{Z}_{p}\right) \rightarrow \operatorname{Gl}\left(n, \mathbb{Z}_{p}\right)$ is a differentiable homomorphism?

\section{References}

[1] H. Bass, M. Lazard et J.-P. Serre, Sous-groupes d'indice fini dans $\operatorname{Sl}(n, \mathbb{Z})$, Bull. Amer. Math. Soc. 70 (1964), 385-392.

[2] V. Belfi and C. Wilkerson, Some examples in the theory of p-completions, Indiana Univ. Math. J. 25 (1976), 565-576.

[3] A. K. Bousfield and D. M. Kan, Homotopy Limits, Completions and Localizations, Lecture Notes in Math. 304, Springer, 1972.

[4] K. S. Brown, Cohomology of Groups, Grad. Texts in Math. 87, Springer, 1982.

[5] M. Conder, A surprising isomorphism, J. Algebra 129 (1990), 494-501.

[6] Y. Félix, S. Halperin and J.-C. Thomas, Rational Homotopy Theory, Grad. Texts in Math. 205, Springer, 2001.

[7] H. Furstenberg, Poisson boundaries and envelopes of discrete groups, Bull. Amer. Math. Soc. 73 (1967), 350-356.

[8] P. de la Harpe, Topics in Geometric Group Theory, Univ. of Chicago Press, 2000.

[9] P. Hilton, On the homotopy groups of the union of spheres, J. London Math. Soc. 30 (1955), 154-172.

[10] P. Hilton, G. Mislin and J. Roitberg, Localization of Nilpotent Groups and Spaces, Notas Mat. 55, North-Holland Math. Stud. 15, North-Holland, 1975.

[11] H. Huang, CUNY doctoral thesis, May 1997.

[12] M. Lazard, Groupes analytiques p-adiques, Inst. Hautes Études Sci. Publ. Math. 26 (1965), 389-603.

[13] C. A. McGibbon, Self maps of projective spaces, Trans. Amer. Math. Soc. 271 (1982), 325-346. 
[14] C. A. McGibbon and J. M. Møller, On spaces with the same $n$-type for all $n$, Topology 31 (1992), 177-201.

[15] - - - On infinite dimensional spaces that are rationally equivalent to a bouquet of spheres, in: Lecture Notes in Math. 1509, Springer, 1992, 285-293.

[16] - - - Connected covers and Neisendorfer's localization theorem, Fund. Math. 152 (1997), 211-230.

[17] C. A. McGibbon and J. Roitberg, Connective coverings, phantom maps and genus sets, Indiana Univ. Math. J. 47 (1998), 1433-1458.

[18] J. L. Mennicke, Finite factor groups of the unimodular group, Ann. of Math. 81 (1965), 31-37.

[19] J. W. Milnor, Topology from the Differentiable Viewpoint, The University Press of Virginia, 1965.

[20] J. M. Møller, The normalizer of the Weyl group, Math. Ann. 294 (1992), 59-80.

[21] J. Neisendorfer, Primary homotopy theory, Mem. Amer. Math. Soc. 25 (1980), no. 232.

[22] —, Localization and connected covers of finite complexes, in: Contemp. Math. 181, Amer. Math. Soc., 1995, 385-390.

[23] D. Rector, Loop structures on the homotopy type of $S^{3}$, in: Lecture Notes in Math. 249, Springer, 1971, 99-105.

[24] J. Roitberg, The genus and SNT sets of certain connective covering spaces: work of Huale Huang, Abstracts Amer. Math. Soc., Meeting \#990, Oct. 11-12, 2003, Abstract \#990-55-76.

[25] J.-P. Serre, Cohomologie des groupes discrets, in: Ann. of Math. Stud. 70, Princeton Univ. Press, 1971, 77-169.

[26] —, A Course in Arithmetic, Grad. Texts in Math. 7, Springer, 1973.

[27] D. Sullivan, Genetics of homotopy theory and the Adams conjecture, Ann. of Math. 100 (1974), 1-79.

[28] M. C. Tamburini, J. S. Wilson and N. Gavioli, On the (2,3)-generation of some classical groups. I, J. Algebra 168 (1994), 353-370.

[29] C. Wilkerson, Classification of spaces of the same n-type for all $n$, Proc. Amer. Math. Soc. 60 (1976), 279-285.

[30] -, Applications of minimal simplicial groups, Topology 15 (1976), 111-130.

IBM Global Services

Morristown, NJ, U.S.A.

Current address of $H$. Huang:

Citigroup

283 King George Rd.

Warren, NJ 07059, U.S.A.

E-mail: huale.huang@citigroup.com
Department of Mathematics \& Statistics Hunter College, CUNY

695 Park Ave., New York, NY 10021, U.S.A. E-mail: roitberg@math.hunter.cuny.edu and

Ph.D. Program in Mathematics The Graduate Center, CUNY 365 Fifth Ave., New York, NY 10036, U.S.A.

Received 29 October 2006; in revised form 28 February 2007 